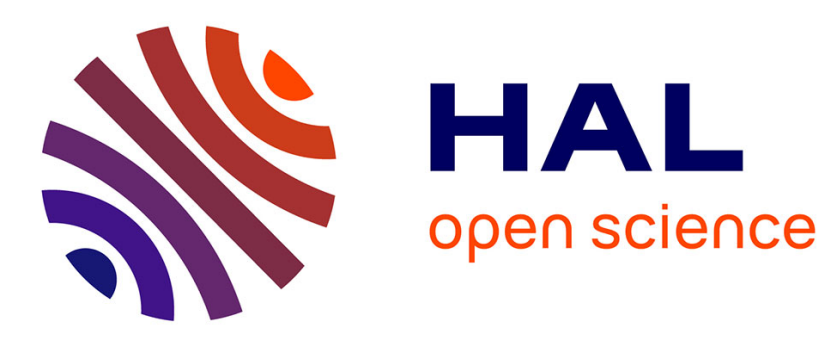

\title{
Analysis and Synthesis of Learning Agent's Communicative Behavior
}

Nik Nailah Binti Abdullah, Stefano A. Cerri

\section{To cite this version:}

Nik Nailah Binti Abdullah, Stefano A. Cerri. Analysis and Synthesis of Learning Agent's Communicative Behavior. Applied Artificial Intelligence, 2005, 19 (9-10), pp.1015-1041. 10.1080/08839510500304116 . lirmm-00105303

\section{HAL Id: lirmm-00105303 https://hal-lirmm.ccsd.cnrs.fr/lirmm-00105303}

Submitted on 11 Oct 2006

HAL is a multi-disciplinary open access archive for the deposit and dissemination of scientific research documents, whether they are published or not. The documents may come from teaching and research institutions in France or abroad, or from public or private research centers.
L'archive ouverte pluridisciplinaire HAL, est destinée au dépôt et à la diffusion de documents scientifiques de niveau recherche, publiés ou non, émanant des établissements d'enseignement et de recherche français ou étrangers, des laboratoires publics ou privés. 


\title{
Analysis and Synthesis of Learning Agent's Communicative behavior
}

\author{
Nik Nailah Binti Abdullah and Stefano A. Cerri \\ LIRMM: CNRS \& Université Montpellier II \\ 161 Rue Ada, Montpellier Cedex 5, France \\ \{binti, cerri\}@ lirmm.fr
}

\begin{abstract}
This paper describes our preliminary results on the induction of human communication protocols for the modeling of Learning Agents in Multiagent Systems based on Cognitive Science studies. The purpose of constructing communication protocols is to support, guide, facilitate, enhance inter and intra organizational communication in Multiagent Systems (MAS). A MAS in turn can support the distributed collaborative problem solving that is required by the GRID by agent communities that dynamically organize themselves having diversified capabilities and needs. Our work has two goals; (i) to infer rules (i.e. protocols) from the communicative behavior of humans in real scenarios and to understand how they learn to infer those rules; (ii) to equip Artificial Agents with those rules and learning mechanism guiding their behaviors, that are somehow similar to the ones adopted by humans. We record sequences of communication exchanges of computer scientists collaborating online as a benchmark for the analysis of regularities that emerge from the exchanges of those communications. We illustrate the findings. Our final objective is to design genuinely autonomous Learning Agents, dynamically adapting to their own experiences. This may represent a major progress in the effectiveness of societies of Distributed Hybrid Systems (human and artificial agents) mutually supporting by delivering services to each other over the GRID.
\end{abstract}

\section{Introduction}

The foundation of our research work is the investigation of problems of communication encountered in real world scenarios; human and MAS. We have considered the problems encountered in human scenarios reviewed by (Clancey 2001) where several scientists collaborate in joint work carried out during the NASA Haughton- 
Mars Project ${ }^{1}$. We have also considered the review of (Hanson et al. 2002) on the MAS scenarios of web service agents for e-business. The center of our study is the what and the why of those communication problems and the how to solve them. The study is then narrowed to analyzing communication protocols among group members in a virtual work practice ${ }^{2}$ environment. We have looked into a particular context: Computer Scientists collaborating online to prepare a deliverable $\mathrm{e}^{3}$ before a given deadline. All communications among collaborating members was carried out over chatting sessions (i.e.BuddySpace) and online meetings (i.e. FlashMeeting ${ }^{4}$ ). Our work involves two stages; i) translating human conversations into $\mathrm{ACL}^{5}$ and ii) learning from these conversations the regularities enabling the induction of communication protocols. We have translated about 40,000 words (i.e. including typing errors and chat jargons) of collaborators conversations into markup ACL messages. We have identified about 4,000 of communicative acts from these exchanges (Binti Abdullah et al. 2005a). We only focus on part (ii) in this paper.

This paper is organized as follows. Section 2 illustrates the real problems encountered in communication scenarios. Section 3 discuss about GRID services and their relationships to learning agents. Section 4 focus on some literature background on the current state of the art in agent communication protocols. Section 5 reflects about learning and communication. Section 6 illustrates the experimental settings. Section 6.1 reviews in detail these findings. Finally, section 7 discusses the conclusions including future work.

\section{Real Scenarios as Motivations}

Hereafter, we review the problems in communication; that had occurred both in human scenarios and MAS scenarios in section 2.1 and 2.2. In section 2.3, we discuss the what and why of these problems.

\subsection{Human scenarios}

(Clancey 2001) reviews scenarios of several scientists collaborating in joint work which was carried out

\footnotetext{
${ }^{1}$ The NASA Haughton-Mars Project (HMP) supports an Exploration program aimed at developing new technologies, strategies, humans factors experience, and field-based operational know-how key to planning the future exploration of the Moon, Mars and other planets by robots and humans. Website: www.arctic-mars.org

${ }^{2}$ Work practice is the collective activity of a group of people who collaborate and communicate, while performing these activities synchronously and asynchronously (Sierhuis et al. 2001).

${ }^{3}$ The deliverable was to be submitted for the Elegi European Union project (IST, VI Framework). Website: www.elegi.org.

${ }^{4}$ BuddySpace (i.e. instant messaging) and FlashMeeting (i.e. video conferencing) are social tools developed by the team at the Kmi, Open University, the UK.

${ }^{5}$ ACL stands for Agent Communication Languages for agents to communicate information and knowledge.
} 
during the NASA Haughton-Mars Project. The group members that were involved are: a mission controller, a mission support, a commander, several crew members, outside experts and occasionally colleagues of the group members. The author analyzed three significant events concerning the communication protocols that had taken place:

Event one The group members had to learn how to use instruments to gather data in the crater to perform a survey of magnetic irregularities. The commander was in regular communication with outside experts, who advised on how particular instruments should be used. During this event, the commander gave a briefing to all his crew members. He gave a lecture on the "aeromag" survey and procedures to follow. After a while, the commander asked to one of the crewmembers who was involved in that procedure if he had read the outside expert's email. The particular crew member replied that he had not read his email yet.

Event two One of the crew members was seeking advice from the outside experts on the safety issue in the hab. He also had a long private discussion with his colleagues. After returning from his private discussion with his colleagues, he requested the group members to a meeting on recommended safety actions to be taken at the hab. The crew member confronted the group with facts and some recommended actions. Each of his reasoning was supported by claims of authoritative outside experts that he had personally received. After some time, the commander tried to get clarifications and explore options, but the crew member became defensive and claimed that the commander had no expertise in the matters at hand, and that they must follow the advice of outside experts. The moment was tense, and very uncomfortable for all of them.

Event three The author (Clancey 2001) conducted a simple experiment with the outside experts to explore the boundaries of what is possible and permissible as communication protocols. NASA paid the Canadian Artic Weather to provide forecasts specific to Haughton Crater. Furthermore, for a fee, the Canadian Artic Weather had to answer questions about weather and the forecasting process to the author. This involves non-operational, scientific advice. The Canadian Artic Weather is requested to answer any questions from the group related issues of weather. Thus, the crew and weather service was engaged in a distributed collaboration with mutual learning. The crew members were allowed to consult outside experts independently, without asking permission from their commanders. This interaction has not proved to be problematic and suggests further that crew members on Mars may be allowed to consult with Earth experts on matters of data interpretation (to be distinguished from operational advice). 


\subsection{MAS scenarios}

Agent1 contacts Agent2 about renting a car (this scenario is adapted from (Hanson et al. 2002)). They engage in a dialogue about Agent1's preferences, Agent2's inventory, and so forth. For protocol, they agree to use a modified Agent Communication Language, in which each message contains standard performatives to identify the intent of the message, and message contents follow a standard car-rental ontology. At some point, Agent1 volunteers the information that it would be willing to pay up to 10 euros more for a convertible. This uses a non-standard performative, so Agent2 replies with "not understood". The negotiation continues as if nothing had taken place. Some time later, Agent2 asks if Agent1 wants a child seat. This term is not defined in the ontology of Agent1. It contacts an external ontology server to find out the term, to be told that it relates to "passengers" who are "children". Agent1 looks in its fact store that there is no information about children in its owner's user profile and no special overrides have been added to this negotiation. So it replies with no thank you. The transaction continues and eventually terminates when both parties are satisfied. This scenario is illustrated in figure 1 below.

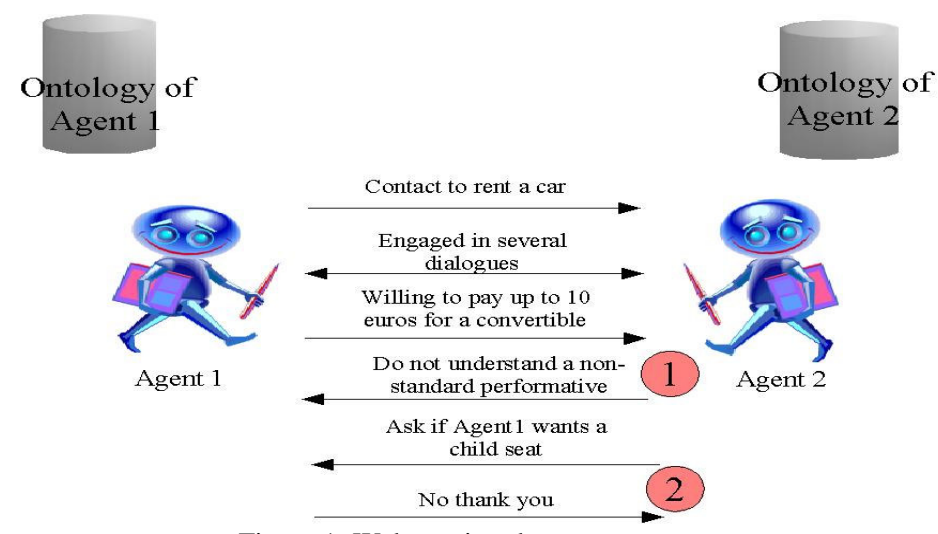

Figure 1: Web services between two agents.

\subsection{Analysis of Human and MAS Scenarios}

Firstly, we will analyze the human scenarios (i.e. Section 2.1). Primarily, we refer to the author's (Clancey 2001) interpretations: 
Event One: If the mission support had been in control, the crew member who had not read his e-mail yet would have received the outside experts' advice; the information would not have come up so haphazardly through the commander during the briefing. The mission controller claimed that all communication should go through mission support, which would then ensure that all members of the crew with a need to know would receive the appropriate information on timely basis. Allowing the mission support to be in control would have prevented the awkward situation that occurred during that briefing.

Event Two: This particular event showed that the communication that had taken place did not worked well. Operations advice, whether it comes from mission support or direct e-mail with a colleague, should only be directed to the commander. An outsider should not be telling a crew member what his/her commander should do. Event Three: This particular event showed that the communication worked very well for all the members. Even if, it violates the mission's controller hypothesis that only the commander should be communicating with outside experts directly.

We now turn to our analytical point of view, illustrating it in figure 2 below:

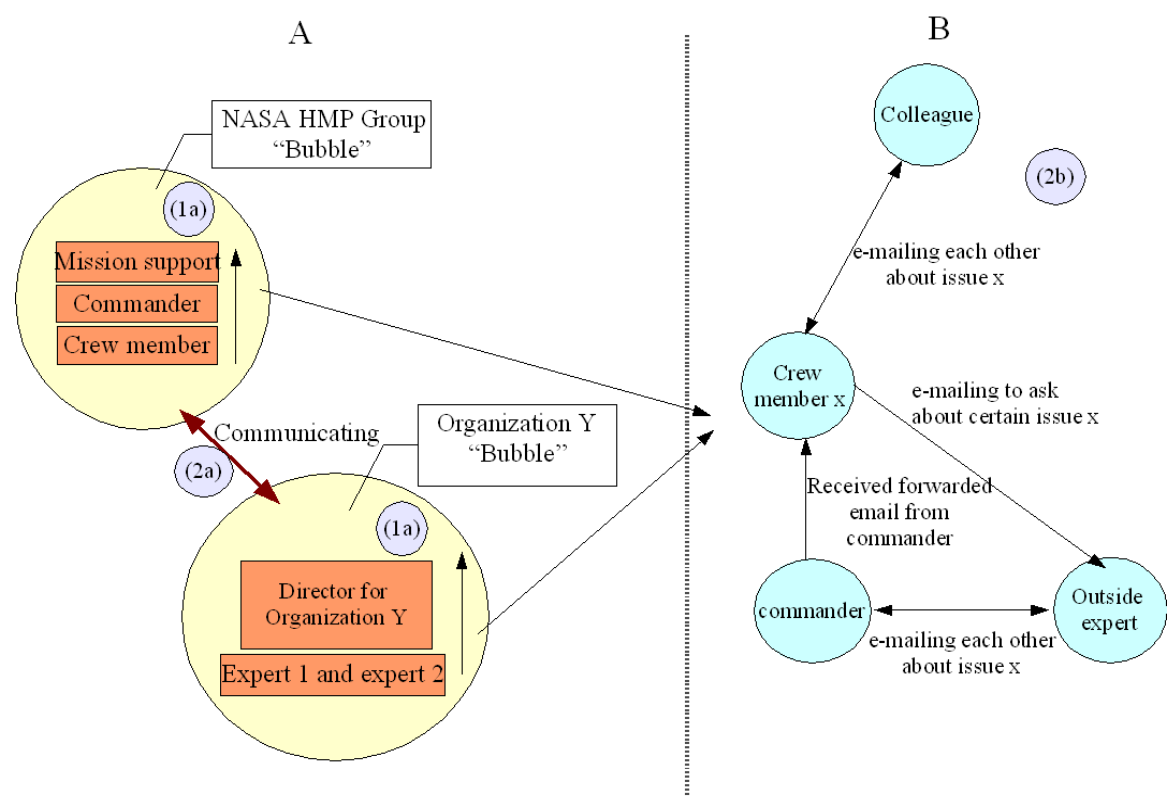

Figure 2: Communications protocols among organizations.

The left hand-side of the figure, denoted by A represent the NASA HMP group and organization $Y$ as existing in their own "bubble". These organizations have their own intentions, goals, and protocols. The right hand side of the figure denoted by B represents the microscopic view of the communication that has taken place. 
What are the major concerns in the above figure 2 ?

1a) Class hierarchy exists in a work practice. Each of the members corresponding to this class hierarchy has also her $^{6}$ own roles. A commander, for example, is in a higher class than crew members. A commander has a role to coordinate his crew members. This is represented by the circle labeled (1a) for the NASA HMP group in figure 4.

2a) Communication efficiency or failure among the internal groups and external groups cannot always be predicted. An example, HMP group members belonging to the NASA organization will not know exactly how to "manage" communication with an external expert coming from another organization for the first time because of the unfamiliarity with the new context they are working with the organization. This is represented by the circle labeled with (2a) in figure 2 .

3a) Any sort of personal crisis among members of the work practice can interrupt or cause problems in decision making and this cannot happen for an important project such as the HMP.

We can associate point (1a) to (3a) to (1b) to (3b): 1b) each member of the crew of NASA HMP has their own duties to carry out. They normally have a guideline that outlines their cooperative and collaborative duties to ensure that they will complete it successfully; 2b) Members of the work practice may work with experts coming from an external society. There must be guidelines that specify what type of communication procedure to use in order to share/exchange information, and to whom in which operation. This is represented by the circle labeled with $(2 b)$ in figure $2 ; 3 b)$ Professional relationships among members of the work practice and people from external society have to be maintained to ensure a good collaboration environment. On the other hand, MAS scenarios (i.e. Section 2.2) concern two important issues: (i) standard performatives defined within the scope of ACL and system failure in recognizing the undefined ones; (ii) pre-defined communication protocols. What do these two scenarios have in common and how do they differ? We examine them in table 1 below:

\footnotetext{
${ }^{6}$ We use her for he/she.
} 
Table 1: The similarities and differences between the scenarios in real world and Multiagent systems.

\begin{tabular}{|c|c|c|c|}
\hline & & Human scenarios (section 2.1) & MAS scenarios (section 2.2) \\
\hline $\begin{array}{l}\text { A)Similar } \\
\text { characteristic }\end{array}$ & $\begin{array}{l}\text { To know what is the } \\
\text { next action to take } \\
\text { in the context of } \\
\text { different situations. }\end{array}$ & $\begin{array}{l}\text { (i) What is the best action to take to } \\
\text { handle this situation? } \\
\text { (ii) Who should I communicate with } \\
\text { to consult prior to taking a decision } \\
\text { for task A? } \\
\text { (iii) Which communication path (i.e. } \\
\text { E-mail, chat, and etc.) is the most } \\
\text { efficient and will be most } \\
\text { appropriate? }\end{array}$ & $\begin{array}{l}\text { (i) I will take this next action in my } \\
\text { protocol. It is the only one specified to } \\
\text { handle this type of situation. }\end{array}$ \\
\hline $\begin{array}{l}\text { B)Different } \\
\text { characteristic }\end{array}$ & To learn. & $\begin{array}{l}\text { (i) This context is almost the same to } \\
\text { the previous context (i.e. I have } \\
\text { learned and induced). I will adapt my } \\
\text { personal rules to this sameness of } \\
\text { context in this communication. }\end{array}$ & $\begin{array}{l}\text { (i) I do not have experience. So I do not } \\
\text { update anything. I am static in my mind. } \\
\text { (ii) I do not understand your meanings. }\end{array}$ \\
\hline
\end{tabular}

In table 1, we list some of the similarities and differences between the humans and agents communication protocols. We can summarize that in $A$ ): (i) both have a set of choices of actions to take. (ii) Humans choose their actions according to the context they are situated in. (iii) Agents choose their actions from their fixed set of protocols. Whereas in $B$ ): (i) how humans select their actions (i.e. behave) is interrelated with their logical type of learning (i.e. private rules) and their transactions with their environments (i.e. contextualizing her role with and in the environment). Humans respond to each other actively according to the context they are situated in (i.e. the whole process of contextualization). They merge communication protocols when learning new experiences, and adapt when they re-encounter similar contexts of situations (Binti Abdullah 2005a,2005b,2005c; Bateson 1972). They are actively contextualizing their processes of learning, merging and adapting. (ii) No such conceptualization of context (see Clancey 1997 on conceptualization and contextualization) nor learning mechanism exists in the agents. Both the real world and agent scenarios are non-deterministic and context dependent.

Defining communication protocols appears to be a difficult task. We assume that eventually in the human scenarios, the group members will communicate efficiently when re-encountering similar context, after having 
learned from their experiences. The three events illustrated an ongoing process of learning, inducing, merging and adapting. The members induced and learned in different situations to adapt to different procedures. Some actions may lead to potential unexpected situations and some may lead to potential good ones. Those unexpected situations become a ground of learning. This in turn, will improve communications in the future. However, in the second scenarios of agents; communication is an actual fact, a static process operating in a dynamic environment. The agents do not have a learning mechanism. Therefore, the agents do not know experience. Since they do not know experience, they cannot compare present to the past. And so, they will not know what is the best action (i.e. what to respond to or how to behave) to take in different context of situations which may be similar to one another to a certain degree. To summarize, the agents will not know how to act in unfamiliar situations. As a result, they cannot handle unexpected messages which may turn out to be valuable. These messages may contain clues as to how they should be handled.

We have explained the what and the why. In the next remaining sections; we will discuss the how. Our study begins at the pragmatics level. We study and analyze the communicative behaviours among collaborators. This research approach is motivated by the work of (Sierhuis et al. 2001). Those authors; (i) represent the people, things and places relevant to the domain and actual behaviour of the people, second by second, over time; (ii) show which of the tools are used when, and by whom to perform certain activities; (iii) include the communication among co-located and distributed people, the communication tools used and the consequences of these communication tools on the practice. (Sierhuis et al. 2001) model human agents to represent how humans act, react and interact with one another and their environment.

\section{GRID Services versus Learning agents}

The authors (Foster et al. 2004) reviewed why the GRID and agents are both concepts and mechanisms for open distributed systems. Readers may refer to their article for further elaboration. Quoting from the paper of Foster et al, "An agent is an encapsulated computer system that is situated in some environment, and that is capable of flexible, autonomous action in that environment in order to meet its design objectives". They are problem solving entities, with well-defined boundaries and interfaces; situated in a particular environment; receive inputs related to the state of their environment; act accordingly through effectors; and autonomous that they have control both over their internal state and over their own behavior (Foster et al. 2004). A successful 
GRID is thus an incorporation of agent communities in MAS. The paper of (Cerri 2002, 2004) proposes that the GRID must consist of technologies allowing autonomous Agents to perform computation and to communicate on the Net in an optimal way. The author emphasizes on the communication and learning of Agent's on the GRID. Since the availability of the Net, the computational process is more and more conversational, between and among "abstract computational entities, human or artificial Agents (Cerri 2004). These agents are able to generate dynamic services to another AA or HA when engaged in conversations (Binti Abdullah et al. 2004). There are 3 different approaches for the modeling of agent communication; $(i)$ human agent $\leftrightarrow$ human agent (HA $\leftrightarrow \mathrm{HA}$ ); (ii) artificial agent $\leftrightarrow$ artificial agent (AA $\leftrightarrow$ AA); and finally (iii) human agent $\leftrightarrow$ artificial agent (HA $\leftrightarrow \mathrm{AA})$. Communication protocols need to be defined in each of these modelling to give a guideline on how agents should communicate with each other and to accommodate the kinds of exceptions that arise in MAS (Paurobally et al. 2003).

\section{Agent Communication Protocols}

Most of the current approaches in the MAS community for modeling agent communication protocols is to predefine the set of actions (i.e. rules) for those agents (Huget et al 2003a; Huget 2003b)). These rules must be able to guide the Agents to handle all types of interaction and any exception during the course of communication. However, as we had reviewed in the previous section, it is highly unlikely to forecast all sequences of actions when agents are communicating on the Web.

We shall review some of the prominent work in modeling communication protocols in MAS. (Pauchet et al. 2003) looks into obtaining experimental protocols which have been analyzed and viewed from human planning and human interactions; to be conceptualized for Artificial Agents. Another work by (Mazouzi et al. 2002) look into evaluating the success of open protocols used by heterogeneous agents; allowing agents to capture and explain the relationships between conversations or group utterances. Considering these major contributions, we believe that the following is missing from their modeling:

i. (Pauchet et al. 2003) design communication protocols based on the subject's actions in a human model of cooperative problem solving based on psychological experiments. However, they do look into how the person, processes and contexts are mutually defining one another and serve aspects of the whole, not as separate elements. 
ii. (Pauchet et al. 2003 and Mazauzi et al. 2002) do not consider how the flow of events from the Agent's perspective, considering self-regulation (i.e. processing content of messages in conformance to her mental states) and oscillation (i.e. integrating change over time).

A person's communication behaviour demonstrates her implicit states of her current activity and how she structures them. Our work is exactly addressing the recognition and interpretation of the internal and context dependent view for the induction of communication protocols. We decided to use human conversations as a benchmark for the analysis of that internal view (i.e. private rules) as well as tracking the social interaction (i.e. adaptation and co-ordination through experience).

\section{Learning and communication}

The foundation of our communication approach is based on the theory of (Bateson 1972). The communication theory of Bateson, deals with the basic understanding of how learning and communication are interrelated with one another. Firstly, learning is categorized into a hierarchy structure following the laws of motion (i.e. rules for describing motion) (Bateson 1972).

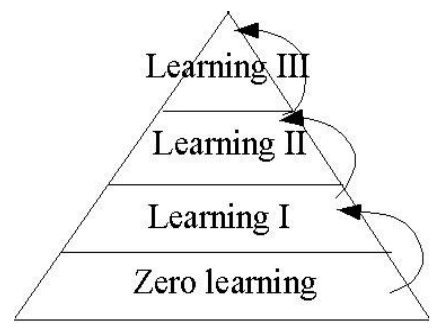

Figure 3: Hierarchy of learning types

Figure 3 illustrates the hierarchy of learning types. The zero learning is the basics of all learning; it is in some degrees stochastic and contains components of trial and error. The curved arrows represent that the one level up in the hierarchy of learning types is described by the motion of change of the level below it. In short, we can summarize it as (i) zero learning: is described by which response is right or wrong and is not subjected to correction; (ii) learning I: is described by the change in the specificity of response by correction of errors of choice within a set of alternatives; (iii) learning $I I$ : is described as the change of process of learning I; a corrective change in the set of alternatives from which choice is made, or it is a change in how the sequence of experience is punctuated; (iv) learning III: is described as the change of process in learning II; a corrective 
change in the system of sets of alternatives from which choice is made. We look only at learning type zero, I and II. Bateson (1972) discusses about the type of learning IV, however we do not illustrate it here as it involves a higher level of learning type that is tied down to evolutionary processes.

The basic elements that distinguish one type of learning from the other are characterized by contexts. As an example, we may have a case in which an entity gives at Time 2 a different response from what it gave at Time 1. This is related to experience, physiology, genetics and mechanical processes (Bateson 1972). These contexts may never be the same, but may have related classes of how a person may respond to it. This stimulus for behaving upon context is then communication. Communication is verbal (i.e. conversations) and non-verbal (i.e. interaction, gestures, and postures). The communication between two persons or among many is described as transaction between the individual and his material and human environment. Quoting from (Bateson 1972):

“...In such systems, involving two or more persons, where most of the important events are postures, actions, or utterances of the living creatures, we note immediately that the stream of events is commonly punctuated into contexts of learning by tacit agreement between the persons regarding the nature of their relationship-or by context markers and the tacit agreement that these context markers shall "mean" the same for both parties."

Are these rules for describing motion corresponding to "communication protocols"? We extend the example given by Bateson (1972). Let us assume in a sequence of events, person A's behavior is perceived as a stimulus for person's B behavior and how person B responds to that behavior is by learning to select from her set of contexts (i.e. all the related events) the next alternatives she takes. In this set of contexts, how does she learn how to know what to respond to that behavior? To answer our posed question above, we refer to these theories; (i) Situated Cognition (Clancey, 1997); (ii) Activity Theory (Leont'ev 1978). Situated cognition is based on the idea that every human thought and action is adapted to the environment that is situated. It looks into; (i) What people perceive (structural view); (ii) How they conceive their activity (functional view) and (iii) What they physically do together (behavioral view). The activity theory on the other hand, emphasizes on what an organism is doing in the world and that the subjectivity of that activity is realized within and constructed by interaction (Clancey 2004). The basic modeling of activity is done in a hierarchical structure with three distinct levels; (i) activitymotive; (ii) action-goal and (iii) operations-condition. Motives gives rise to the need of activity, and actions are basic components of activities. A goal of an action is a conscious goal that guides the action. Finally the operations are ways of executing actions in that they are concrete conditions required to achieve the goals. (i) 
and (ii) will help to explain (iii) learning and (iv) communication. It can explain how humans induce communication protocols by relating it to how a person coordinates (i.e.(i)) as a whole by conceptualizing her context (i.e.(i),(iii)) of what her activity is (i.e. (i),(ii)) when communicating (i.e. iv,(i)) structured by her private rules (Binti Abdullah et al. 2005b).

\section{Experimental Settings}

This section illustrates our experimental settings. We had recorded daily chats and meetings for the period of 7 months. We kept track of Phil's and Mack's communication as our subjects for analyzing communication protocols, for several reasons: (i) Phil is the project manager for a joint project and he is new to this environment (i.e. instant messaging and video-conferencing); and (ii) Mack, on the other hand, is an experienced collaborator and has run many virtual collaboration sessions. We show samples of the environments below in figure 4 and 5 .

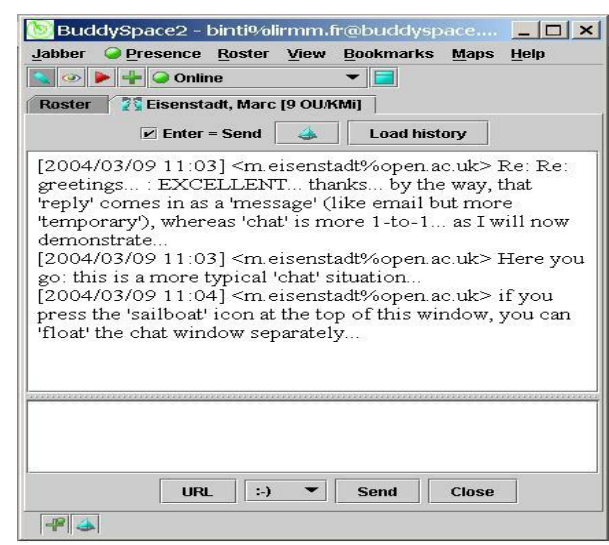

Figure 4: Daily chat between two group members

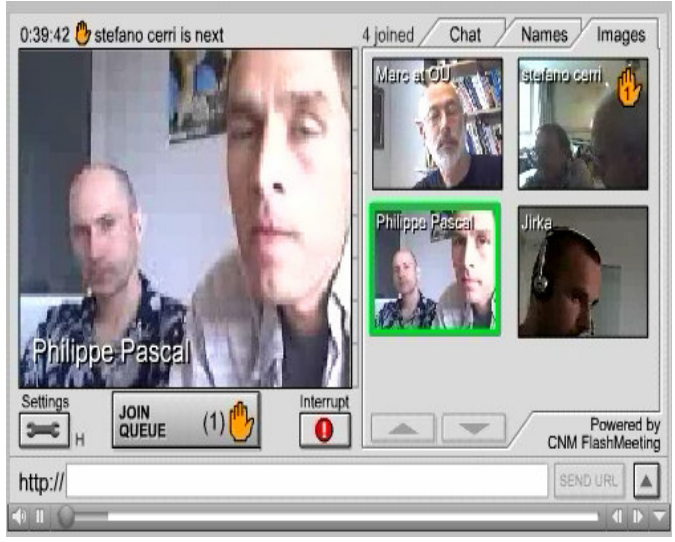

Figure 5: A typical virtual meeting

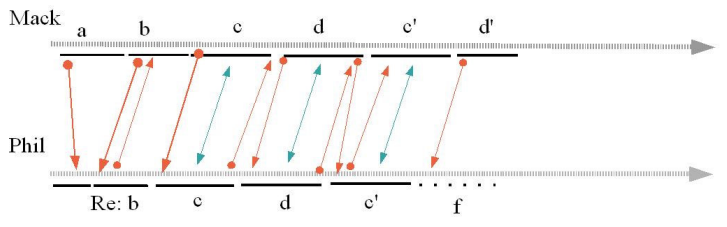

$\mathrm{a}=1-2-1$ chat
$\mathrm{b}=$ broadcasting
$\mathrm{re}-\mathrm{b}=$ reply to broadcasting
$\mathrm{c}=$ tutorial
$\mathrm{d}=$ debugging
$\mathrm{c}^{\prime}=$ tutorial 2
$\mathrm{~d}^{\prime}=$ debugging 2

Figure 6: Observing the transitional states of the collaborators. 
Figure 6 corresponds to figure 4 . It is the representation that we have used to identify where and how Phil had induced communication protocols. Activities are labeled as a,b,c and d. c' is a similar type of activity to c and so is d' to d. The arrows denote who changed the context of communication. The double directed arrows denote the exchanges of communication. The horizontal line denotes the time of activity. We illustrate the steps for identifying changes that had occurred during the transitional states: (i) Locate the point of changes of activities. (ii) Look at what are the events that had caused the activity to take a change. (iii) Locate at which events A's behavior was responding in a way similar to B's. (iv) Compare the communication structures (i.e. conversations or conversations and interaction with tools) of A (i.e. Phil's) and then compare it to B (i.e. Mack's). (v) Now, compare the communication structures of A's to any of her previous set of related events (i.e. contexts). (vi) Study the differences and then generalize the changes. (vii) Continue for related behaviors.

\subsection{Preliminary results on the induction of communication protocols}

In this section, we discuss a particular finding where Phil had communicated in a way similar to how one of his collaborators had communicated. As discussed in section 6 of our method for recognizing changes, we compare this particular conversations structure of Phil's to that collaborator. After doing so, we find similar contexts of communication (i.e. giving instructions, starting a meeting) of Phil's. We then distinguish the differences and similiarities between the conversations structure at context 1 at time 1 to the conversations structure at context 2 at time 2 and so on. We comment the results of our interpretation of the analysis below.

Context $\mathrm{c}_{1}$ : Phil is giving instructions to start a meeting on the 17/09/2004.

"Good afternoon everybody. I am Phil from .... and we are sized together online, maybe we could just start the meeting. So I shall start......,

Context $c_{2}$ : Phil is giving instructions to a newcomer to the environment on how to use the features of the tool on the $21 / 09 / 2004$.

“Good afternoon Simon. Maybe it is your first time practicing this kind of meeting. So there is 1 button to start and to stop broadcasting and to join the queue. So you have to press to start and to ask for the queue and to stop broadcasting as well".

We show in table 2 the converted conversations of Phil during context $c_{1}$ and $c_{2}$.

\footnotetext{
${ }^{7}$ The complete conversations are not shown to protect the privacy of the individuals and the ongoing project.
} 
Table 2: Sample of the converted conversations of Phil during two similar context.

\begin{tabular}{|l||l|}
\hline Context $\mathbf{c}_{\mathbf{1}}$ & Context $\mathbf{c}_{\mathbf{2}}$ \\
\hline greet $\mathrm{p}$, all, $\mathrm{u}$ & greet $\mathrm{U}$ \\
\hline inform $\mathrm{p}$, all $(\mathrm{am})(\mathrm{ph})(\mathrm{lirmm})$ & inform $\mathrm{p}, \mathrm{sm}(\mathrm{prc})(\mathrm{mtg})(1 \mathrm{stm})^{\wedge}(\mathrm{t}-\mathrm{is})(1)(\mathrm{bt})$ \\
\hline inform-ref p,all (szd) (us)(online)(nw) & $\begin{array}{l}\text { inform-ref } \mathrm{p}, \mathrm{sm} \\
\text { (bt)(str) (brdc) }\end{array}$ \\
\hline
\end{tabular}

The full conversion can be referred to the Annex. We abstract the conversation structures to clearly demonstrate the changes. We denote the communicative acts as symbols. Refer to table 3 below.

Table 3: Symbols used to represent the communicative acts

\begin{tabular}{|l|l|l|l|l|}
\hline $\mathrm{g}=$ greet & $\mathrm{c}=$ confirm & $\mathrm{cfp}=$ call for proposal & ir=inform-ref & $\mathrm{i}=$ inform \\
\cline { 1 - 3 } \cline { 1 - 2 }$=$ request when & rwv=request whenever & & &
\end{tabular}

We only represent the communicative acts with their primitives (see table 4 , we do not represent $p$, all in this example) for analyzing the conversation structures.

Table 4: The abstract representation of the conversation structures

\begin{tabular}{|l|l|}
\hline Converted conversations & Abstract representations \\
\hline greet $\mathrm{p}$, all, $\mathrm{u}$ & $\mathrm{g} \mathrm{U}$ \\
\hline inform $\mathrm{p}$, all $(\mathrm{am})(\mathrm{ph})(\mathrm{lirmm})$ & $\mathrm{i}(\mathrm{y} 1)(\mathrm{y} 2)(\mathrm{y} 3)$ \\
\hline
\end{tabular}

Below, in figure 7 and 8, we illustrate the flow of sequences of the conversation structures of Phil, during both context $\mathrm{c}_{1}$ and $\mathrm{c}_{2}$ respectively.

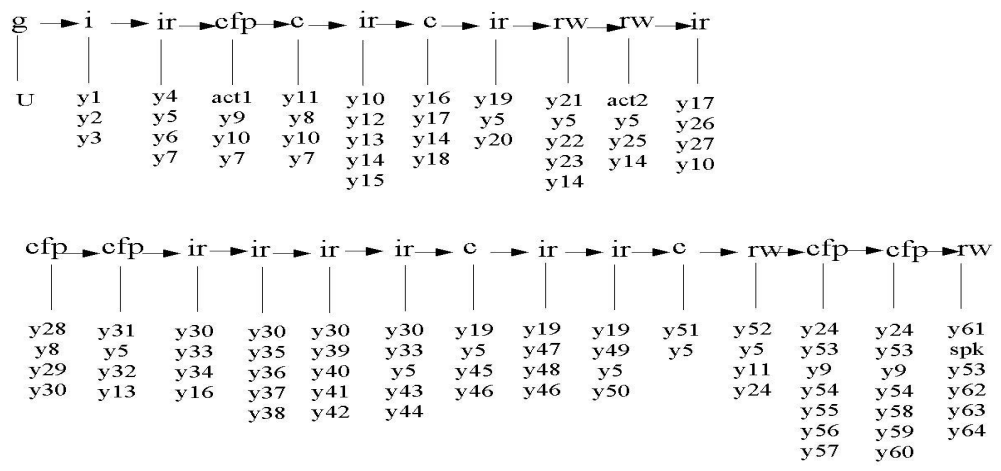

Figure 7: The sequences of the conversation of Phil during context $c_{1}$.

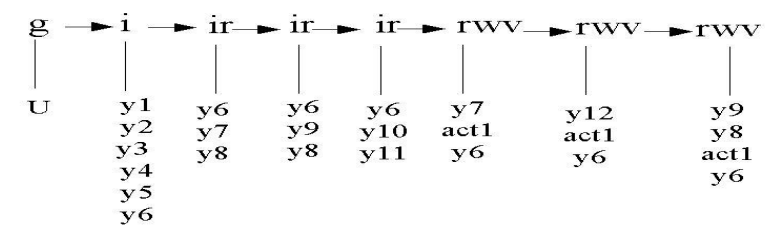

Figure 8: The sequences of the conversations of Phil during context $c_{2}$. 
Referring to table 2 of the first and second column, they corresponds to figure 7 and figure 8 respectively. They are the illustrations of the conversation sequences of Phil's during two similar ${ }^{8}$ contexts. It is to illustrate how the communicative acts along with the primitives have been re-sequenced. The $y_{x}$ of figure 8 and 9 are not similar primitives. We relate this analysis and synthesis to learning and communication theory (i.e. as discussed in section 5) and the analysis of section 2.3. This analysis involves two major cases: (i) Phil's private rules (i.e. figure 8); (ii) the adaptation and merging of protocol; improvised by induction; generalizing and then conceptualizing the description (i.e. figure 9). We illustrate the differences: (i) The communicative act $r w$ (i.e. request when), $c f p$ (i.e. call for proposal) and $c$ (i.e. confirm) is not communicated during context $\mathrm{c}_{2}$; (ii) The communicative act $r w v$ (i.e. request whenever) is not communicated during context $c_{1}$. We illustrate the similarities: (i) Both communications begins with the communicative act $g$ (i.e. greet), has ir (i.e. inform-ref). We illustrate the flow of the sequences: (i) The communicative acts ir (i.e. inform-ref) does not precede $i$ (i.e. inform), and follows immediately after $i, c f p, r w$ and $c$; (ii) in $\mathrm{c}_{2}$, the sequences of conversations of Phil's has been has been re-enacted and re-sequenced ${ }^{9}$ when compared to his other sequences ${ }^{10}$. In this particular context $c_{2}$, Phil had adapted and merged the protocols of Mack's (i.e. from the observation of how Mack had handled the situation) into his own private rules when he re-encountered similar situation.

Firstly, from the differences of $(i)$ and (ii); we make a hypothesis that the selection of communicative acts is contextualized and influenced by the mental states of the speaker. For example A) request when (i.e. j) is communicated to request the listener to perform an action as soon he believes in the precondition. On the other hand, $B$ ) the communicative act request whenever (i.e. f) is communicated to request the listener to perform the same set of actions whenever her preconditions is fulfilled. A) is a context that request temporary respond during that ongoing discussion (i.e. inform me during this meeting what we must do). B) is a context such that it requests that at anytime a similar context appears, the partner should respond in a similar way (i.e. whenever you have something to say when using this tool, please take this action).

Secondly, from the flow of the sequences of $(i)$ and $(i i)$ we notice the following. The sequences are $r e$ sequenced and then re-enacted accordingly to how the individual is contextualizing. Inform-ref (i.e. b) is used frequently: (i) to give description to an object; (ii) to give description of the request made; (iii) to give

\footnotetext{
${ }^{8}$ We use this notation to represent the sameness of context of y at time 1 and context of $y$ at time 2 . The context y cannot be of the same at time 1 and time 2 .

${ }^{9}$ This particular analysis is not illustrated in this paper. Readers may refer to (Binti Abdullah et al. 2005a and 2005b).
} 
description of support for the call for proposal; (iv) to give description of support to the uncertainty of a certain knowledge of an object. This is quite an obvious analysis. It can be influenced by: (i) the reflection of the reasoning of the speaker. This reflection again is dependent on the subject which the speaker wishes to communicate about; or (ii) learned experiences. What are the processes involved in this selection of communication protocols (i.e. communicative acts and its primitives) and the re-enacting and re-sequencing of it? Our approach to this is to identify the inter-relationships terms used in Cognitive Science as discussed in (Clancey 1997). This will allow us to define the processes involved.

(i) Context: Is a conception of what a person is doing, and hence the context of her actions. It is always social.

(ii) Experience: Is related to contextualism ${ }^{11}$ that experience consists of events. The interaction of the person and the physical relations provide support for the experiences. The relations are then analyzed into textures. These textures lying in context. Time is involved in shaping this experience.

(iii) Events: Is defined as having a quality as a whole. Quality is defined as the total meaning of the event.

(iv) Situations: Situations is a pointer for re-encountering the set of context and is partially constructed within the interpretation process.

(v) Learning zero, I and II: The unidentified operators responsible for the changes of the learning types (i.e. refer to figure 3, section 5) as reported by (Bateson 1972).

An experience is learning of an event, and remembering this event when re-encountering situations resembling the previous learned events. This process changes in time. What is exactly a context then? It is not a simple variable that is manipulated. It is constructed by the subject, in an ongoing manner (Clancey 1997). We use the notion contextualizing and context inter-dependently. Contextualizing is a whole process of coordination (i.e. from the social and biological perspective). Context is then the lying ground of events. A set of contexts is thus defined as set of events having a certain degree of similarity among one another. The learning involved is inter-related to the stimulus-response (the notion of stimulus-response here do not treat stimulus-response as a simple case of manipulation) on the early stimulus-response theory) behavior of humans. In particular, we refer to Bateson (1972) for the stimulus-response abstraction. We give an example where we have person A and person $\mathrm{B}$ responding to each other based on this idea. We have $a_{1} b_{1} a_{2} b_{2} a_{3} b_{3} a_{4} b_{4} a_{5} b_{5}$ where the $a$ 's refer to

\footnotetext{
${ }^{11}$ Contextualism is a term associating to "actively doing something all the time". Its other name is pragmatism, and has roots in William James, C.S Pierce and John Dewey (Clancey 1997).
} 
the items of A's behavior, and the b's refer to the items of B's behavior. We represent the speaker (i.e. who is currently talking) and listener (i.e. who is currently listening) as A and B respectively. We represent Phil as B in below's model.

(i) $\left(a_{i} b_{i} a_{i+1}\right)$, in which $a_{i}$ is the stimulus for $b_{i}$ (i.e. A's behavior is the stimulus of Phil's behavior); (ii) $\left(b_{i-1} a_{i} b_{i}\right)$, in which $a_{i}$ is the response to $b_{i-1}$, which response B reinforces with $b_{i}$ (i.e. A's behavior is the response to Phil's previous behavior which response of Phil's reinforce with the current behavior); (iii) $\left(a_{i-1} b_{i-1} a_{i}\right)$, in which $a_{i}$ is now A's reinforcement of B's $b_{i-1}$, which was response to $a_{i-1}$ (i.e. A's behavior is A's reinforcement of Phil's previous behavior).

Bateson had also suggested three basic ideas; stimulus, context of stimulus, context of context of stimulus and so on:

(i) Stimulus; which is defined as the elementary signal that may be internal or external. We represent it as a function $f^{S i}$ and $f^{S e}$ respectively and $f^{S}$ is a summation of both functions. An internal stimulus may be represented as task or a goal. An external stimulus is represented as the inputs coming from the environment (i.e. person's B's behavior is a stimulus for person's A behavior); (ii) Context of stimulus; which is defined as a metamessage which classifies the elementary signal. We represent it with function $f^{C s}$; (iii) Context of context of stimulus that is a meta-metamessage which classifies the metamessage and so on. This requires a decomposition of function of functions.

To demonstrate the modelization of the terms in relationship to one another, we give an example below.

Scenario 3: Unexpected goal.

Line 1:Greg: "Hi! How is it going? Everything is fine?"

Line 2:Peter: "Yes! I am in the middle of wrapping up the article for the conference on our projects"

Line 3:Greg: "Aha!"

Line 4:Greg: "Would you be interested to use this particular map in that paper, the one that I showed to you in Prague?"

Line 5:Peter: "Indeed!"

Line 6:Greg: "Hang on..I should be able to push it in this consortium..give me some minutes..."

Line 7:Greg: "Just need 5 minutes..."

These are conversations between Greg and Peter, who are both coming from different organizations. They are collaborating on a joint project together. Figure 10 below is the conceptualization of Greg's stimulus-response 
behavior in relationship to events, and context.

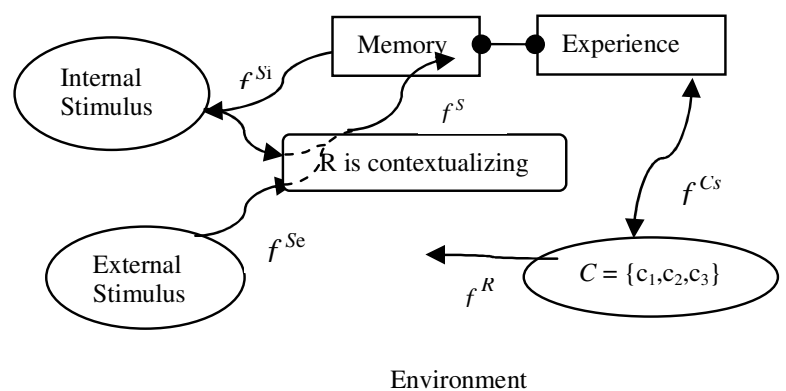

Figure 10: The conceptualization of Greg's behavior with its processes.

Figure 10 is a naïve view of how we illustrate the process. These functions representing the processes that are responsible for the re-enacting and re-sequencing of communication protocols. Referring to figure 10 , Greg is always actively contextualizing himself in a society. What influences his contextualization is related to the functions which we describe below:

$$
\begin{gathered}
\left(\left(f^{S \mathrm{e}} \vee f^{S \mathrm{i}}\right) \vee\left(f^{S \mathrm{e}} \rightarrow f^{S \mathrm{i}}\right)\right) \rightarrow f^{S}(\mathrm{Eq}) 1 \\
\left(f^{S} \circ f^{C s}\right) \rightarrow f^{R} \quad \text { (Eq) } 2
\end{gathered}
$$

(Eq) 1 states that there could be either a stimulus coming from the society (i.e. $f^{S \mathrm{e}}$ ) or an internal stimulus (i.e.f ${ }^{\mathrm{Si}}$ ). An external stimulus on the other hand can trigger internal stimulus. Here, Greg is asking Peter how he is without having the intention of proposing to Peter of using the map in the first place. However, when Peter mentioned about the article, he suddenly remembers, (or it can probably fulfill partly one of his goal) that he can provide some information in that article of Peter (i.e. referring to scenario 3, page 18, at line 2,3 and 4 ). Therefore, the stimulus could be both, responding to someone's previous behavior and at the same having a predefined goal. Stimulus coming from the environment and/or internal have some role in the communication response. Referring to line 4, Greg had also reminded Peter of a previous event where he had shown the map to him at Prague. The stimulus here is also coupled to remembering of past events from his experiences. Now, this disjunction of function is then mapped as a compound stimulus function $f^{S}$. (Eq) 2 states that the altered processes that had taken place in $(\mathrm{Eq}) 1 ; f^{S}$ is now composed with another process $f^{C s}$. We visualize the $f^{C s}$ contains another function (i.e. process) that discriminate and generalize contexts to select the next actions 
denoted by a class $C=\left\{\mathrm{c}_{1}, \mathrm{c}_{2}, \mathrm{c}_{3}\right\}$. We give an example: $\mathrm{c}_{1}=$ proposing to someone to include chapter 1 in their joint article $\mathrm{c}_{2}=$ proposing another person to have a meeting; $\mathrm{c}_{3}=$ proposing to have a meeting using the instant messaging.

Now, we have $f^{R}$ is the function composition (i.e. $f^{S}$ and $f^{C s}$ ) trigger responses back into the society (i.e. hence as how the individual is socializing himself in a society). As a result, all this processes occuring as a whole, during the contextualization (there are more processes involved during the contextualization, this is only a fraction of it). We represent the response as function to incorporate the function for calling some procedures from the activity states framework (Binti Abdullah et al 2005c).

The function must be able to handle changes of processes; the mapping from one process to the other. Again we stress, the value manipulated as argued above, are not simply variables. Recognizing what sort of functions that represents the process processes is another issue to be deal after. To summarize this section, we make two assumptions:

(i) Contextualizing involves multiple process that discriminate and generalize. So we need to find out what are the processes involved for discriminating and generalizing. As example; I learn that this situation is similar to the previous one based on my previous experiences (i.e. remembering). I shall respond in this way (i.e. apply this communication protocols).

(ii) Learning of new communication protocols involves the merging and adaptation of others (i.e. from experiences or individuals when re-ecountering similar situations) to our own private rules.

\section{Conclusions and perspectives}

The activity of learning has faculties such as imagination, conceptualization, reasoning, comparing, remembering, confirming and conviction. It is a consultation of human discourse or interaction and integration among all kinds of entities. Induction is one of the characteristics of human reasoning, that we form generalization based on our experience or observations. Through observations and experiences one learns. When humans experience certain situations, they learn and keep that in memory. These experiences are learned over time. In short, from our analysis, we found that human (i) have private rules; (ii) learn from experience; (iii) private rules and learning may be monitored, modelled and used in real contexts (i.e. for Learning Agents in 
Multiagent System). People apply their own protocols and induce, merge and adapt new ones when communicating with one another in a new environment when they re-encounter similar contexts; adapting to experience (i.e. learning). When they re-encounter similar situations, they are in fact inducing situations which are to them similar to the previous ones by remembering. How those internal changes of process of merging and adapting is influenced by their private rules (i.e. manner of behaving or communicating). Particularly what is interesting is even if the communication protocols are adapted to context that are similars to the ones experienced, their private rules are never actually changed. When communicating in non-related contexts, they return to communicate in their own manner. Only in the experienced contexts, do they made slight modifications in their communication protocols.

Now, we discuss about the perspectives of our future work. Our conceptualization of the stimulusresponse behavior is far from completion. We have not considered for now: (i) how does the individual perceives her activities; (ii) how to incorporate situations and events into the stimulus-response functions; (iii) how to model the discrimination and generalization; (iii) how is the selection being made to know what to respond with (i.e. sameness of context); (iv) how to model the merging and adaptation of communication protocols. A very important issue is: how does one remember? How do we represent Agents to remember past events? Events and contexts seem to play a significant role for the adaptation and merging of communication protocols. It will be wrong to make an assumption that there is a repeatable context at all times (i.e. context at time 1 is equivalent to context at time 2), quoting from Bateson (Bateson 1972). If one assumes so, then there would only be one type of learning that is learning not subjected to correction (i.e. basic stimulus-action modeling). Secondly, we have not analyzed the sequences of conversations when one is engaged in multiple topics. Thirdly, we have not consider that in human situations, there are the perception and senses. In an agent's world, where agents are on the GRID, do not have perception. This makes it more difficult to fully realize the autonomy of Agents. We have to distinguish certain processes that can be deal with and others that we cannot deal with. Fourthly, what exactly are the learning operators that had enable humans to discriminate and generalize? Are these operators in relation with the claims that humans merge and adapt communication protocols? And if so, how does one know which context is "similar" to the previous context? These highly context dependent norms (i.e. rules) are exactly the major objective of our investigation. The realization of autonomous artificial agents will not be possible until they will not be equipped with rules guiding their behaviors that are somehow similar to the ones adopted by humans in similar contexts. Similar means inspire: we are totally aware of the differences. We are far from 
defending any anthropomorphic model of Artificial Agents, rather, in the long term; we wish to get inspired by Human Agent's conversational behavior in order to progressively augment Artificial Agent's autonomy in social behavior, thus their adequateness to real communication scenarios.

\section{Acknowledgments}

We would like to extend our appreciation to Maxim Makatchev, research programmer of the Learning and Research Development Center, University of Pittsburgh, the US; for his contribution in commenting the work from the application point of view. Not forgetting Sylvain Degeilh of Computational Linguistic, LIRMM:CNRS \& Universite Montpellier II, France; for his contribution in commenting on the formalism of the conversations. Also to Philippe Lemoisson of LIRMM:CNRS \& Universite Montpellier II, France; Professor Marc Eisenstadt of KMI, Open University, the UK; and Professor Joost Breuker of SWI, University of Amsterdam, the Netherlands for their cooperation.

\section{References}

Binti Abdullah, N.N., Liquiere, M., Cerri, S.A. 2004. Steps towards the induction of communication protocols: GRID Learning Agents. In $1^{\text {st }}$ International Workshop on GRID Learning Services, GLS'04, (pp:64-77), Maceio, Brazil.

Binti Abdullah, N.N., and Cerri, S.A. 2005a. Formalizing Human Conversations : Steps to the induction of communication protocols. Submitted to the International Joint Conference in Artificial Intelligence (IJCAI2005), Edinburgh, Scotland.

Binti Abdullah, N.N., and Cerri, S.A. 2005b. Some preliminary results on the: Steps to the induction of communication protocols. Submitted to the Cognitive Science 27 ${ }^{\text {th }}$ Annual Meeting (CogSci2005), Stresa, Italy.

Binti Abdullah, N.N and Cerri, S.A. 2005c. Communicative Acts Selection Based on Activity States: The First Step Towards the Induction of Communication Protocols. Submitted to the Agent Communication Workshop, The fourth international joint conference on Autonomous Agents and Multiagent Systems (AAMAS05). Utrecht, The Netherlands.

Bateson, G. 1972. Steps to an ecology of mind. Chandler and Publications Co. 
Cerri, S.A. 2002. Human and Artificial Agent's Conversations on the GRID. $1^{\text {st }}$ LeGE-WG International Workshop on e-Learning and GRID Technologies: Educational Models for GRID based services. Lausanne, Switzerland.

Cerri, S.A. 2004. An integrated view of GRID services, Agents and Human Learning. (In press). IOS Press Book.

Clancey, W.J. 1997. Situated Cognition:On Human Knowledge and Computer Representations. Cambridge University Press.

Clancey, W.J. 2001. What we are learning about communication protocols. Link: http:// bill.clancey.name

Clancey, W.J. 2004. Simulating Activities: Relating Motives, Deliberation, and Attentive Coordination. In Cognitive Systems Research, special issue "Situated and Embodied Cognition” 34(2) 125-137

Ferber, J. 1999. Multi Agent Systems: An Introduction to Distributed Artificial Intelligence. Addison Wesley Longman, Harlow UK.

FIPA-ACL 2002. FIPA-ACL Communicative Acts Specifications. In the Foundation for Intelligent Physical Agents Repository Online.

Foster, I., Jennings, N.R., and Kesselman, C. 2004. Brain Meets Brawn: Why Grid and Agents Need Each Other. Proceedings of the Autonomous Agents and Multiagent Systems Conference (AAMAS 2004), ACM press. Pp: $8-15$.

Hanson, J.E, P. Nandi and D.W. Levine. 2002. Conversation-enabled Web Services for Agents and e- business. Proceedings of the International Conference on Internet Computing (IC-02), CSREA Press. June 24-27. pp: 791-796.

Huget, M.P., \& Koning, J.L. 2003a. Interaction Protocol Engineering. In the Communications in Multiagent Sytems, Agent Communication Languages and Conversation Policies. Ed. Marc-Philippe Huget. Springer Verlag Publications. LNAI 2650, pp:179-193

Huget, M.P (2003b) Design Agent Interaction as a Service to Agents. In the Communications in Multiagent Sytems, Agent Communication Languages and Conversation Policies. Ed. Marc-Philippe Huget. Springer Verlag Publications. LNAI 2650, pp:209-222.

Leont'ev, A.N. 1978. The problem of Activity and Psychology. Philosophy in the USSR, Problems of Dialectical Materialism. Progress Publishers 
